\title{
10 SERVIÇOS DE ATENDIMENTO MÓVEL DE URGÊNCIA FRENTE ÀS EMERGÊNCIAS PSIQUIÁTRICAS: UMA REVISÃO NARRATIVA
}

\author{
| Alana dos Santos de Souza ${ }^{1}$; Helena Moraes Cortes²; Paula Hayasi Pinho ${ }^{3}$ |
}

\section{RESUMO}

CONTEXTO: O atendimento na crise pode ser realizado pelos: Centros de Atenção Psicossocial, emergências dos hospitais gerais, urgência/ emergência dos pronto-socorros, atenção primária à saúde e os Serviços de Atendimento Móvel de Urgência.

OBJETIVO: Realizou-se uma revisão narrativa sobre os serviços de atendimento móvel de urgência frente às emergências psiquiátricas.

MÉTODO: Foram realizadas buscas de artigos nas bases de dados: SCIELO, BVS, CAPES e LILACS publicados entre 2013 a 2018 . Para a busca dos artigos utilizou-se as seguintes palavras-chaves: emergências psiquiátricas, crise psíquica, serviço de atendimento móvel de urgência no atendimento à crise, sendo identificados 23 estudos.

RESULTADOS: Observou-se uma escassez de estudos sobre os serviços de atendimento móvel de urgência frente às emergências psiquiátricas. Os serviços de urgência e emergência, quando prestam assistência à pessoa em crise psíquica, tem priorizado a sedação e o encaminhamento para o internamento hospitalar, reproduzindo o saber da psiquiatria tradicional. A dificuldade dos profissionais de saúde em atender a pessoa em situações de urgência e ou emergência psiquiátrica, por vezes está relacionada com a deficiente formação, e com a falta de educação permanente que abarque a saúde mental.

CONCLUSÃO: O cuidado prestado pelo Serviço de Atendimento Móvel de Urgência à pessoa em sofrimento psíquico é fundamental para o fortalecimento da reestruturação da assistência à saúde mental no país. É necessário o treinamento das equipes que atuam nesses serviços para garantir a função substitutiva dos serviços da Rede de Atenção Psicossocial e, o cuidado das pessoas que vivem e convivem com transtornos mentais fora das internações hospitalares.

\section{PALAVRAS-CHAVE: Saúde mental; Intervenção na crise; Serviços de saúde mental}

\section{RESUMEN}

\section{"Servicios de atención móvil de urgencia frente las emergencias psiquiátricas: Una revisión narrativa"}

CONTEXTO: La atención a la crisis puede ser realizada por: Centros de Atención Psicosocial, emergencias de los hospitales generales, puertas hospitalarias de atención a la urgencia / socorro, atención primaria a la salud y los Servicios de Atención Móvil de Urgencia.

OBJETIVO: Se realizó una revisión narrativa sobre los servicios de atención móvil de urgencia frente a las emergencias psiquiátricas.

MÉTODO: Se realizaron búsquedas de artículos en las bases de datos: SCIELO, BVS, CAPES y LILACS publicados entre 2013 a 2018.En la búsqueda de los artículos se utilizaron las siguientes palabras clave: emergencias psiquiátricas, crisis psíquica, servicio de atención móvil de los servicios de atención telefónica la urgencia en la atención a la crisis, siendo identificados 23 estudios.

RESULTADOS: Se observa la escasez de estudios sobre los servicios de atención móvil de urgencia frente a las emergencias psiquiátricas. Los servicios de urgencia y emergencia, cuando prestan asistencia al usuario en crisis psíquica, han priorizado la sedación y el encaminamiento para la internación hospitalaria, reproduciendo el saber de la psiquiatría tradicional. La dificultad de los profesionales de la salud en atender a la persona en situaciones de urgencia y o emergencia psiquiátrica, a veces está relacionada con la deficiente formación, y con la falta de educación permanente que abarque la salud mental.

CONCLUSIÓN: La asistencia prestada por el Servicio de Atención Móvil de Urgencia a la persona en sufrimiento psíquico es fundamental para el fortalecimiento de la reestructuración de la asistencia a la salud mental en el país, es necesario el entrenamiento de los equipos que actúan en esos servicios para garantizar la función sustitutiva de los servicios de la salud Red de Atención Psicosocial y el cuidado de las personas que viven y conviven con trastornos mentales fuera de las internaciones hospitalarias.

\section{DESCRIPTORES: Salud mental; Intervención em la crisis; Ser- vicios e salud mental}

\begin{abstract}
"Moving services of urgency in front of psychiatric emergencies: A narrative review"

BACKGROUND: Crisis care can be carried out by: Psychosocial Care Centers, general hospital emergencies, hospital emergency care / emergency room doors, primary health care and Mobile Emergency Care Services.

AIM: To carry out a narrative review on emergency mobile services in the face of psychiatric emergencies.

METHODS: Articles were searched in databases: SCIELO, VHL, CAPES and LILACS published between 2013 to 2018. The following keywords were used to search for the articles: psychiatric emergencies, psychic crisis, mobile urgency in dealing with the crisis, with 23 studies being identified.

RESULTS: There is a shortage of studies on emergency mobile services in the face of psychiatric emergencies. The emergency and emergency services, when assisting the user in psychic crisis, have prioritized sedation and referral for hospital admission, reproducing the knowledge of traditional psychiatry. The difficulty of health professionals in caring for the person in situations of emergency and or psychiatric emergency is sometimes related to poor training and lack of permanent education that includes mental health. CONCLUSION: The assistance provided by the Mobile Emergency Service to the person suffering from mental illness is fundamental to strengthen the restructuring of mental health care in the country, it is necessary to train the teams that work in these services to guarantee the substitutive function of the services of the Psychosocial Attention Network and the care of people who live and coexist with mental disorders outside hospital admissions.
\end{abstract}

\section{KEYWORDS: Mental health; Crisis intervention; Mental health services}

Submetido em 31-03-2018

Aceite em 09-07-2018

1 Estudante do $9{ }^{\circ}$ semestre de Enfermagem na Universidade Federal do Recôncavo da Bahia, Centro de Ciências da Saúde, Bahia, Brasil, alanasanttos94@gmail.com

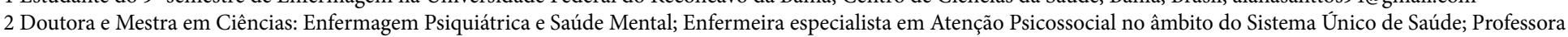
Assistente na Universidade Federal do Recôncavo da Bahia, 44570-000 Cajueiro - Santo Antônio de Jesus - Bahia, Brasil, helena@ufrb.edu.br

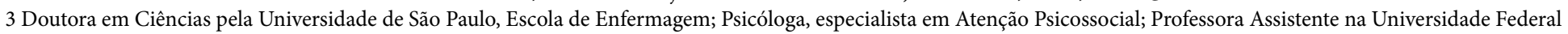
do Recôncavo da Bahia, 44570-000 Cajueiro - Santo Antônio de Jesus - Bahia, Brasil, paulahpinho@gmail.com

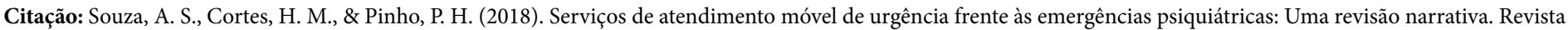
Portuguesa de Enfermagem de Saúde Mental (20), 72-80. doi: 10.19131/rpesm.0229 


\section{INTRODUÇÃO}

O perfil epidemiológico das emergências psiquiátricas, tem passado por mudanças no decorrer das últimas décadas, pela razão da Reforma Psiquiátrica brasileira. As emergências psiquiátricas são caracterizadas por situações de crise psíquica que os usuários apresentam, embora, nem toda a situação de crise determine uma emergência, entretanto, estas estão intimamente relacionadas (Bonfada, 2010; Quevedo e Carvalho, 2014). Para a psiquiatria tradicional a crise é entendida como uma situação de grave desequilíbrio resultante da doença mental. Entende-se como uma agudização e exacerbação do adoecer mental, e tem como consequências ações que objetivam a contenção e a redução dos sintomas, por considerar que o funcionamento geral do indivíduo está prejudicado e, este pode se tornar incapaz de assumir responsabilidades pessoais (Amarante, 2007; Willrich, 2016).

Em contrapartida, no campo da atenção psicossocial a crise também é compreendida como uma situação de sofrimento psíquico intenso, que produz uma desorganização mental, e pode ser originada de aspectos ligados ao convívio familiar e social do indivíduo, proporcionando o desligamento da sua realidade, principalmente no meio social, afastando-se, restritamente, do sentido limitado da agudização sintomatológica (Amarante, 2007; Willrich, 2016).

A Reforma Psiquiátrica brasileira, procurou substituir o modelo de assistência psiquiátrica de tipo manicomial, por uma rede de serviços substitutivos de apoio à saúde mental e psiquiatria. $\mathrm{O}$ cuidado à saúde na perspectiva da atenção psicossocial, vem se consolidando e mostrando-se resolutivo. Apesar disso, a atenção à saúde precisa ser ampla e considerar o sujeito na sua integralidade, e não apenas o transtorno psíquico por ele apresentado (Demarco, Jardim e Kantorski, 2016). O movimento de desinstitucionalização no Brasil, fundamentado na Reforma Psiquiátrica, visou a reestruturação da assistência à saúde mental no país, procurando a integração das pessoas com transtornos mentais, no seu meio social, por meio da valorização de serviços estratégicos, comunitários e de territorialização, bem como novas formas de cuidados e, a garantia do direito à cidadania, desconstruindo o estigma da loucura (Maftum, Silva, Borba, Brusamarello e Czarnobay, 2017).

Os serviços de assistência substitutivos do modelo manicomial no atendimento à crise são prestados pelos componentes integrados da Rede de atenção Psicossocial (RAPS).
$\mathrm{O}$ atendimento na crise pode ser realizado pelos: Centros de Atenção Psicossocial (CAPS) nas suas diferentes modalidades, emergências dos hospitais gerais, atenção à urgência/emergência nos prontos socorros, atenção primária à saúde por meio das Unidades Básicas de Saúde (UBS) e, os Serviços de Atendimento Móvel de Urgência (SAMU) (Brasil, 2011).

Para o Ministério da Saúde do Brasil, não há local específico e, exclusivamente responsável, para atender às situações de crise, nem sendo tampouco de exclusividade dos profissionais de saúde. $\mathrm{O}$ atendimento à crise deve acontecer no lugar onde se encontra o indivíduo, ou seja, no seu circuito de vida ou de cuidado: residência, via pública, serviços de saúde como UBS, CAPS, pronto-socorro, entre outros lugares de convívio em que o indivíduo se encontre (Ministério da Saúde, 2013).

Diante disso, os serviços de urgência e emergência assumem um lugar importante no atendimento às pessoas com transtornos psíquicos. O SAMU deve prestar atendimento inicial à pessoa em crise psíquica em domicílio, em via pública, ou quando precise ser transferida de uma UBS, para o atendimento especializado em saúde mental, podendo ser este preferencialmente o CAPS, ou os hospitais gerais (Campos, 2015).

Desse modo, entendemos que para o atendimento à pessoa em situação de crise psíquica é necessário que hajam profissionais capacitados, desde o atendimento de emergência até ao encaminhamento para o serviço especializado, visando minimizar os riscos de complicações para o indivíduo e para outros, sendo necessário que os profissionais saibam lidar com essa situação, para conseguir trabalhar na estabilização do quadro e recuperação do sujeito.

Mesmo que a equipe de urgência/emergência esteja preparada para atuar em situações de crise, é comum se deparar algumas vezes, com a carência de conhecimentos específicos, visto que no currículo acadêmico das universidades em geral, o atendimento à emergência em saúde mental é abordado de forma breve, principalmente nos cursos técnicos, tornando-se um problema de saúde de difícil abordagem para as equipes, em diversas categorias (Brito, Ramos, Arruda, Dias e Silva, 2013).

O imaginário social de que o doente mental oferece periculosidade, o estigma, o preconceito e o desconhecimento revelam-se como dificuldades de acesso para pessoas em situações agudas aos serviços do Sistema Único de Saúde (SUS) de maneira geral. 
Em virtude disso, verifica-se a necessidade de qualificação das práticas de cuidado por meio do desenvolvimento das competências e, da literacia em saúde mental e psiquiatria dos cuidadores, nas situações de crise psíquica em serviços de emergências (Ministério da Saúde, 2016).

A partir das problemáticas apresentadas, fica evidente a necessidade de conhecer a atuação dos profissionais de saúde diante de uma situação de crise psíquica. Nesse contexto, emerge a seguinte questão de pesquisa: qual o entendimento dos profissionais que atuam no Serviço de Atendimento móvel de Urgência sobre o atendimento à crise psíquica?

\section{MÉTODOS}

Trata-se de um estudo qualitativo de revisão narrativa da literatura. Para a elaboração deste trabalho utilizouse uma pesquisa bibliográfica em estudos primários, descritivos, exploratórios e de abordagem qualitativa e quantitativa, por meio da análise de artigos científicos e teses, disponíveis em base de dados eletrônicos de acesso livre (SCIELO, BVS, CAPES e LILACS) publicados nos últimos cinco anos, entre 2013 a 2018. Para a busca dos artigos nas bases de dados utilizou-se as seguintes palavras-chaves: emergências psiquiátricas, crise psíquica, serviço de atendimento móvel de urgência no atendimento à crise.

Os critérios de inclusão foram: artigos que abordassem a crise no paradigma da atenção psicossocial e, a atuação do serviço de atendimento móvel de urgência, editados em língua portuguesa e publicados nos últimos cinco anos. Ao final da pesquisa, foram selecionados 23 artigos nas bases de dados consultadas. Os resultados surgiram após serem lidos os resumos e, selecionados os que mais se aproximavam do objeto de estudo. Os resultados foram analisados pelo método comparativo com a literatura científica.

\section{RESULTADOS E DISCUSSÃO}

Os resultados foram agrupados em três categorias empíricas, a saber: a crise frente ao paradigma da atenção psicossocial; desafios dos profissionais de saúde no atendimento à crise na perspectiva da atenção psicossocial; o serviço de atendimento móvel de urgência na assistência à crise psíquica.

\section{A Crise Frente ao Paradigma da Atenção Psicossocial}

A loucura sempre existiu na nossa sociedade, sendo vista de diferentes maneiras no decorrer das épocas.
O modo como a loucura era (é) considerada pela sociedade, estabelecia o tratamento e a forma de cuidado a ser prestado. Atualmente, as pessoas que possuem transtornos psíquicos são cuidadas a partir de uma nova compreensão do adoecer mental, que não é de cura, mas sim de suporte, pois o foco não é mais a doença (Demarco, Jardim e Kantorski, 2016).

Para compreendermos o conceito do processo de saúde e do adoecer mental é necessário refletir e, considerar que as pessoas estão sujeitas a diversas condições que, podem levar a desenvolver transtornos mentais em algum momento da vida - como os fatores físicos, ambientais, sociais, culturais, econômicos, entre outros (Paes e Maftum, 2013). As emergências psiquiátricas ou crises podem ser conceituadas como alterações agudas do pensamento, do comportamento e do humor, causadas por transtornos psiquiátricos ou crise psicológica, a qual implica uma intervenção terapêutica imediata, em consequência de ser capaz de haver evolução rápida para um resultado danoso, tanto individual quanto social (Martins, H. Neto, R. Neto e Velasco, 2015; B. Sadock, V. Sadock e Ruiz, 2016).

Para a psiquiatria clássica, a crise é entendida como uma situação na qual há disfunção grave, que ocorre exclusivamente no decurso da doença. Devido a esse entendimento, a resolução para a crise baseia-se em conter a agressividade, conservando ações que eram realizadas no quotidiano dos manicómios e que acabou por marginalizar e excluir as pessoas em sofrimento psíquico da sociedade (Willrich, Kantorski, Chiavagatti, Cortes e Antonacci, 2013). Na lógica da atenção psicossocial, a crise é compreendida como expressão existencial, em resposta as situações produzidas nas relações do contexto de vida social e familiar do sujeito. Por isso, esta é considerada como condição social mais do que biológica, e requer dos serviços de atenção psicossocial a responsabilização, por meio de acolhimento e a construção de vínculos afetivos dos profissionais em relação às pessoas que estão sendo cuidadas (Willrich et al, 2013).

De acordo com Almeida, Nascimento, Rodrigues e Schweitzer (2014), as pessoas em grave sofrimento psíquico passam por condições críticas, desencadeando os momentos de crise, e passam a reproduzir ações confusas e incompreendidas pela própria pessoa e, por aqueles que estão próximos. Essas ações podem levar à necessidade de avaliação e intervenção dos serviços de emergência. Para Brito, Bonfada e Guimarães (2015), nem toda situação de crise psíquica determina absolutamente uma urgência ou emergência em saúde mental. 
Se tratarmos toda a crise como urgência ou emergência, seguimos reproduzindo intolerância e preconceito com as pessoas com transtornos psíquicos, e acabamos por demonstrar a incapacidade de compreender e aceitar estas pessoas no nosso dia-a-dia, reproduzindo o saber da psiquiatria tradicional, limitando a pessoa à doença. Os momentos de manifestações da crise psíquica por uma pessoa são vistos por muitos como uma situação urgente, quando começa a afetar o quotidiano interpessoal. Na maioria dos casos, as pessoas sentem-se incomodadas ou ameaçadas pelo comportamento do indivíduo em sofrimento psíquico, e isto acaba por determinar, quando há necessidade de chamar os profissionais de assistência à urgência e emergência (Brito, Bonfada e Guimarães, 2015).

Alguns dos sentimentos mais comuns numa pessoa em situação de crise evidencia-se pela incerteza, ameaça e insegurança, tanto para o indivíduo em crise quanto para os que convivem com ele, produzindo uma configuração social, marcada por severas dificuldades de comunicação entre os envolvidos, o que termina por ampliar o problema e gerar uma percepção de urgência. Portanto, esses momentos devem ser compreendidos por profissionais qualificados que precisam acolher o indivíduo nas suas especificidades (Ministério da Saúde, 2013).

Corroborando com Almeida et al. (2014) e Oliveira, Ferreira, Vasconcelos e Dutra (2017), na lógica da atenção psicossocial, valoriza-se a subjetividade, o saber e o sentir do sujeito numa situação de crise, com o propósito de compreender o que está acontecendo com ele. Procuram-se novas formas de cuidados, como por exemplo, a escuta qualificada, e o atendimento aos familiares e/ou acompanhantes, a estabilização do quadro, o estabelecimento de hipótese diagnóstica, a exclusão de causa orgânica, e encaminhamento para a continuidade do cuidado nos serviços da Rede de Atenção Psicossocial.

A assistência em saúde mental, no serviço de Atenção Psicossocial, requer uma relação de parceria no cuidado entre o profissional, o utente e a família; para que o cuidado prestado seja mais eficiente, com o propósito de que a família compreenda e aceite a pessoa com transtorno mental, participando ativamente, e contribua para a melhoria do quadro.

Além disso, é fundamental conhecer melhor a pessoa em sofrimento psíquico e, ajudar a equipe a redimensionar-se de acordo com as necessidades que são exigidas.
Dessa forma, a relação utente e profissional, é uma experiência em que ambos podem desenvolver capacidades interpessoais e, ajudar na compreensão do problema psíquico do sujeito (Fernandes, Pereira, Leal, Sales e Silva, 2016).

\section{Desafios dos Profissionais de Saúde no Atendimento à Crise na Perspectiva da Atenção Psicossocial}

Segundo Santos (2013, p.15), “a assistência à saúde mental no Brasil, encontra dificuldades em relação a implantação da reabilitação psicossocial/inserção social frente ao estigma social, à doença mental e à pessoa com transtorno mental". É bastante comum, a inexperiência de aproximação da população em geral e dos profissionais de saúde a uma pessoa em situação de crise, em consequência dos séculos de isolamento e exclusão, aos quais vem sendo submetidas as pessoas que vivem e convivem com transtornos mentais (Ministério da Saúde, 2013).

Ao longo da história, a sociedade tem lidado com a loucura excluindo as pessoas com transtornos mentais do seu quotidiano.

Os hospitais psiquiátricos têm mantido o papel de isolar as pessoas em sofrimento psíquico, alegando oferecer tratamento para melhorar a saúde dessas pessoas. Entretanto, sabe-se das situações de violência e de degradação, que as pessoas internadas nos hospitais psiquiátricos sofreram e tem sofrido. Diante desse contexto, as pessoas em sofrimento psíquico são estigmatizadas também pelos profissionais de saúde, e muitas condutas ainda são baseadas no modelo biomédico (Holanda, Eduardo, Silva, Nascimento e Flavio, 2016; Salles e Barros, 2013).

Mesmo com a reestruturação da assistência à saúde mental no país, muitos ainda vêem as pessoas com transtornos psíquicos, principalmente nos seus momentos de crises, como alguém perigoso.

Para Bastos, Dutra, C. Silva, Pacheco e T. Silva (2016), mesmo depois de quarenta anos do início dos movimentos pela Reforma Psiquiátrica e suas conquistas, percebe-se que alguns profissionais ainda preservam práticas do pensamento manicomial. O estigma, a doença mental, as concepções erróneas e a falta de formação e de treino das equipes de saúde, tornam-se fontes de insegurança para muitos profissionais quando se deparam com usuários agitados e/ou agressivos (Quevedo e Carvalho, 2014). 
O Serviço de Atendimento Móvel de Urgência na Assistência à Crise Psíquica

A partir do século XVIII, o cuidado às pessoas com transtornos psíquicos era prestado pelos manicómios, sendo restrito ao internamento. Esse modelo de atenção à saúde das pessoas com transtornos mentais, aos longos dos anos, era a única opção existente e, referência de tratamento em saúde mental. Os manicómios eram instituições de exclusão e estabeleciam que a loucura precisava de ser isolada (Coneglian, Cavalcante e Leandro, 2015). A RAPS, no Brasil, tem por objetivo ampliar o acesso dos serviços à população em geral e responsabilizar-se pela atenção à saúde das pessoas com transtornos mentais e com necessidades de uso de crack, álcool e outras drogas, e as suas famílias. Está no bojo da RAPS, a articulação do cuidado entre os pontos da rede de base territorial, permitindo o desenvolvimento do vínculo dos utentes e qualidade do cuidado por meio de acolhimento, do acompanhamento, da atenção nas urgências, tendo o internamento como último recurso terapêutico (Cortes, 2016).

O SAMU, conforme a Portaria no 3.088/2011 é um dos serviços de atenção às urgências/emergências em saúde mental, compondo a RAPS, em substituição ao atendimento de urgência/emergência nos hospitais psiquiátricos; logo, tem papel fundamental no cuidado das pessoas em sofrimento psíquico intenso e, com necessidades decorrentes do uso de álcool e outras drogas (Brasil, 2011). As urgências e emergências ocupam um lugar estratégico nos cuidados, consideradas portas de entradas para os usuários com transtorno mental, em situação de crise, nos serviços do SUS. Entretanto, tem se apresentado como um dos pontos problemáticos da RAPS, em razão de que na maioria das vezes, os serviços de urgência e emergência, quando prestam assistência ao utente em crise psíquica, tem priorizado a sedação e o encaminhamento para o internamento hospitalar, como se estes métodos fossem a principal e única possibilidade para conter a crise (Oliveira e Silva, 2017; Brito, Bonfada e Guimarães, 2015).

$A$ assistência prestada pelo SAMU à pessoa em sofrimento psíquico é fundamental para o fortalecimento da reestruturação da assistência à saúde mental no país. Mas, segundo Almeida et al. (2014), o seu modelo de atenção é baseado no paradigma biomédico, associando, muitas vezes, a visão de que as emergências em saúde mental estão relacionadas com a agressividade, e a hiperatividade, conduzindo o entendimento de crise para a descompensação psicopatológica.
Desta forma, buscando de imediato a estabilização do quadro clínico, por meios de contenção mecânica ou química, por diagnósticos e, doses medicamentosas definidas em protocolos.

As ações de contenção mecânica ou química têm a finalidade de evitar que os utentes atuem de forma violenta durante os momentos de agitação, e somente deverão ser utilizadas, quando práticas não coercitivas, como o diálogo e a escuta, não forem suficientes para lidar com expressões de agressividade (Willrich, Kantorski, Antonacci, Cortes e Chiavagatti, 2014). Os serviços de emergências têm o histórico de desconhecer ou não atuar de forma que a atenção à crise psíquica necessita, ou seja, por meios da empatia, do diálogo, da humanização, da corresponsabilização pelo cuidado e da criatividade durante o atendimento ao indivíduo em situação de crise (Oliveira e Silva, 2017). Zeller \& Citrome (2016), apontaram os benefícios das práticas não coercitivas no atendimento a crise pelos serviços de emergências.

Estas incluem a redução de lesões físicas e psicológicas para os utentes, bem como a redução do uso de recursos, custos e além de melhorar a relação entre profissionais de saúde e utente. Os autores ressaltam que para muitos utentes pode ser a sua primeira experiência no serviço de saúde mental, de forma negativa; o que pode influenciar em não cooperação dos utentes com os profissionais de saúde e, comprometer assim, os atendimentos futuros.

De acordo com Bastos et al (2016), em seu estudo sobre saúde mental no atendimento pré-hospitalar móvel, observaram que ainda existe a percepção dos profissionais do SAMU de que os fluxos dos utentes atendidos, por esse serviço, possuem direcionamento prioritário para a rede hospitalar, não articulando intervenções acompanhadas de outros serviços de base territorial à saúde mental. Isso pode contribuir para fragilizar os serviços da Rede de Atenção Psicossocial.

Santos et al (2014), evidenciaram noutro estudo, que dos 18.820 atendimentos realizados pelo SAMU, no período em estudo, 5,2\% foram prestados a indivíduos com quadros de urgências psiquiátricas, e que a maioria dos atendimentos foram prestados no domicílio das pessoas assistidas, 46,6\% foram contidas e transportadas para o hospital psiquiátrico de referência, $81,2 \%$ são feitos com auxílio da polícia militar, $87,5 \%$ dos profissionais têm dificuldades no atendimento psiquiátrico e, $58,4 \%$ requerem formação e treino em saúde mental e psiquiatria. 
Ainda conforme estes autores, observou-se que 58,3\% dos profissionais, desconheciam os princípios da Reforma Psiquiátrica e a legislação referente à Política Nacional de Saúde Mental (PNSM), 81,5\% desconheciam as estratégias utilizadas pelo serviço, para atuar conforme as diretrizes da PNSM. Nesse contexto, os sujeitos conceituaram as urgências e emergências psiquiátricas como as situações onde o utente predispõe ameaça à vida a si e a outros, confirmando o status de periculosidade, atribuído ao sujeito em crise (Santos et al, 2014). Daggenvoorde, Gijsman \& Goossens (2017), buscaram explorar experiências de utentes e suas famílias, no primeiro contato de emergências por uma equipe móvel de atendimento à crise. Os relatos dos utentes demonstraram que eles se sentiram estigmatizados, como criminosos, quando houve envolvimento da polícia para garantir a segurança no atendimento. Tal abordagem também foi apontada como difícil de ser aceita pelos seus familiares. Conforme o estudo, para os profissionais que realizaram o primeiro contato com os sujeitos em crise, ainda há pouca orientação sobre como interagir nestas situações.

Bonfada, Guimarães, Miranda e Brito (2013), perceberam noutro estudo que, os profissionais de saúde demonstraram compreensões que contradizem a Reforma Psiquiátrica brasileira. Os autores evidenciaram que os profissionais que atuam no SAMU não dão credibilidade ao modo da atenção psicossocial; os discursos dos profissionais revelaram que, eles entendem que os utentes de saúde mental necessitam de internamento psiquiátrico.

Nesse contexto, observa-se que o modelo baseado na psiquiatria tradicional ainda permanece, no ideário dos profissionais de saúde como referência para a assistência psiquiátrica.

Para Buedo \& Fiadaron (2016), o internamento é considerado um recurso terapêutico de natureza restritiva e, só pode ser realizada quando proporcionar maiores benefícios, do que as intervenções que podem ser realizadas no meio familiar, comunitário e social. Segundo Daggenvoorde, Gijsman \& Goossens (2017), a avaliação no domicílio e, o tratamento nos serviços comunitários precisa ser a primeira escolha a ser considerada, quando possível, antes de recomendar a admissão numa unidade de internamento para um sujeito em crise. Segundo Almeida et al. (2015), as diretrizes do SAMU não informam os conteúdos das formações e treinos das equipes.
Isso abre oportunidade para que os profissionais atuem com base nas práticas da psiquiatria tradicional, e tendo a transferência do utente para o internamento como única opção de assistência à crise psíquica, divergindo do preconizado pela atenção psicossocial.

Brito et al (2013), apresentaram no seu estudo que apesar de vários avanços na assistência à saúde mental, ainda há uma grande dificuldade dos profissionais que atuam no serviço do SAMU, em prestar assistência de qualidade. E isso, pode estar relacionado com as características e, comportamentos que os utentes apresentam em situações de emergências psiquiátricas, e essas dificuldades podem estar relacionadas com o estigma, o desconhecimento sobre o assunto ou desinteresse dos profissionais. O medo foi citado por $90 \%$ da equipe, como um fator que dificulta prestar a assistência. Entretanto, a empatia coloca o profissional como facilitador do atendimento, visto que este tem um olhar para o utente, observando a sua integralidade e, podendo assim prestar uma assistência de maior qualidade.

Conforme Bonfada et al (2013), diante da deficiência dos serviços estratégicos para atenção à saúde mental e psiquiatria, as famílias dos utentes usam o SAMU como uma possibilidade para o internamento perante os momentos de crise. Os argumentos dos profissionais entrevistados revelaram que muitas vezes, o internamento acontece devido à incapacidade do familiar em conviver com o doente mental, diante da singularidade que, o indivíduo em sofrimento psíquico apresenta. Entretanto, os autores ressaltam que não se deve atribuir toda a responsabilidade à família pois, esta deve ser considerada como parceira no acompanhamento terapêutico, e não como cuidadora exclusiva dos utentes com transtornos psíquicos grave.

O internamento pode desvalorizar o usuário e estabelecer a ideia de que as pessoas com transtornos mentais são incapazes de construir o seu quotidiano fora das instituições.

Para o Ministério da Saúde, o internamento precisa de ser considerado como último recurso e não como solução imediata à crise psíquica. Devendo também, quando necessário, ser realizada prioritariamente nos CAPS III (funcionamento por 24 horas), com a lógica do acolhimento integral, e nos hospitais gerais de forma articulada com a rede (Salles e Barros, 2013; Ministério da Saúde, 2013). Os profissionais de saúde, quando decidirem pelo internamento como um recurso terapêutico nas situações de crise, devem assistir o utente na sua integralidade. 
É preciso levar em consideração, a necessidade de manter os laços sociais e familiares, bem como, a continuidade do tratamento para serviços de apoio territorial. Além do que, a família deve participar e acompanhar todo o processo de hospitalização do sujeito (Buedo \& Fiadaron, 2016).

De acordo Silva e Dimenstein (2014), a atenção à crise revela-se como um centro estratégico do cuidado da RAPS, visto que, o seu cuidado fora dos internamentos dos manicómios, garante a permanência dos utentes no seu meio familiar e comunitário. $\mathrm{O}$ que pode possibilitar a territorialização e a globalização do cuidado, interrompendo o trajeto do internamento como forma de segregação. A atenção à crise, sendo desse modo realizada, garante a função substitutiva dos serviços e as ações da RAPS. As autoras ressaltam que, a qualificação das práticas e dos serviços são elementos imprescindíveis à sustentabilidade da Reforma Psiquiátrica.

Segundo Oliveira e Silva (2017), no que se refere ao SAMU, os treinos das equipes, é instituído e definido pela Portaria 2.048/GM, assim, realiza-se uma capacitação dos profissionais, no momento em que o serviço é implantado através dos municípios. E durante essa capacitação, somente são dedicadas duas horas de formação às intervenções de saúde mental. Sendo este momento exclusivamente teórico, sem nenhuma abordagem prática. A concepção do SAMU, como um serviço de atendimento que precisa intervir rápido e com pontualidade, impossibilita uma ação ampliada das equipes à subjetividade que os utentes apresentam. As ações estão direcionadas para as estratégias preestabelecidas, sistematizadas, protocoladas, que dificultam ações subjetivas. Em virtude disso, este serviço ratifica a crise no modo da psiquiatria tradicional, como simples exacerbação sintomatológica e, principalmente caracterizada pelo risco que os utentes podem oferecer, entendendo que, as situações de crise precisam ser controladas e solucionadas imediatamente (Almeida et al., 2015).

Almeida et al. (2015), enfatizam que para que ocorra uma mudança nas práticas assistenciais da saúde mental e psiquiatria, torna-se necessário superar o modelo tradicional da psiquiatria, rediscutir o conceito da loucura na sociedade e, repensar o modo das práticas assistenciais, a partir do paradigma da atenção psicossocial e dos princípios do SUS. Nesta perspectiva, concordamos com Amarante (2007), quando diz que é preciso mudar mentalidades, mudar as atitudes, e mudar as relações sociais.

\section{CONCLUSÕES}

Os resultados apresentados nesta revisão permitiram identificar que a assistência prestada pelo serviço de atendimento móvel de urgência, no Brasil, às pessoas em situação de crise psíquica, tem como principal desfecho o encaminhamento para as unidades hospitalares.

É essencial, que os profissionais que atuam no SAMU durante o atendimento a uma pessoa em crise psíquica, tenham a compreensão de que vários aspectos podem ocasionar um desequilíbrio mental, desde uma descompensação psicopatológica até os fatores de stress ou estressores da vida quotidiana. Dessa forma, é fundamental procurar ter uma definição clara do problema, através da formulação dos diagnósticos de enfermagem, da implementação de intervenções, que impliquem o utente e a família como parceira, o diálogo e a escuta qualificada. Esta compreensão pode contribuir, para que estes profissionais saibam lidar com o comportamento e, a situação psicossocial do utente em crise e da sua família.

É necessário que, as equipes tenham a definição clara dos papeis de cada profissional durante o atendimento na crise.

Caso haja a necessidade de uma contenção e internamento temporário, o utente deverá ser encaminhado prioritariamente para o CAPS, conforme prevê a portaria da RAPS. É imprescindível que, a rede de serviço de saúde do SUS, seja acompanhada de profissionais que saibam atender a todas as situações envolvendo os utentes na sua integralidade e, que possam dar os encaminhamentos adequados para os determinados problemas de saúde.

A educação permanente das equipes multiprofissionais que atuam no SAMU é fundamental para garantir a função substitutiva dos serviços da RAPS e, o cuidado das pessoas que vivem e convivem com transtornos mentais, fora dos internamentos hospitalares. Torna-se necessário a educação permanente, afim de instrumentalizá-las quanto aos atendimentos e, encaminhamentos adequados para os demais serviços integrantes da RAPS.

Assim, considera-se fundamental que as equipes que atuam nesses pontos da rede, estejam receptivas e motivadas para as atividades de educação permanente, baseadas nos cuidados preconizados pelo modo de atenção psicossocial. 


\section{REFERÊNCIAS BIBLIOGRÁFICAS}

Almeida , A., Nascimento, E., Rodrigues, J., e Schweitzer, G. (2014). Intervenção nas situações de crise psíquica: dificuldades e sugestões de uma equipe de atenção pré-hospitalar. Revista brasileira de enfermagem, 708-714. Disponível em: http://www.scielo.br/scielo. php?pid=S0034-71672014000500708\&script=sci_abstract

Almeida, A., Nascimento, E., Rodrigues, J., Zeferino, M. T., Souza, A. e Vieira.P (2015). Atendimento móvel de urgência na crise psíquica e o paradigma psicossocial. Revista Texto e Contexto Enfermagem, 24 (4). 1035-1043. Disponível em: http:// www.scielo.br/scielo.php?script $=$ sci_arttext $\&$ pid $=$ S0104-07072015000401035

Amarante, P. (2007). Saúde Mental e Atenção Psicossocial. Rio de Janeiro: Fiocruz.

Bastos, F., Dutra, C., Silva, J. A., Pacheco, K., e Silva, T. (2016). Saúde Mental No Atendimento Pré- hospitalar Móvel. Revista Portuguesa de Saúde Mental, (spe 4), 17-23. Doi: 10.19131/rpesm.0136

Bonfada, D. (2010). Serviço de Atendimento móvel de urgência (SAMU) a assistência ás urgências psiquiátricas. (Dissertação Mestrado). Universidade Federal do Rio Grande do Norte, Brasil.

Bonfada, D., Guimarães, J., Miranda, F. A., e Brito, A. A. (2013). Reforma Psiquiátrica Brasileira: Conhecimentos Dos Profissionais De Saúde Do Serviço De Atendimento Móvel De Urgência. Revista Escola Ana Nery, 17 (2), 227-233. Doi: 10.1590/S1414-81452013000200004

Brasil. Ministério da Saúde. (2011). Portaria No 3.088, de 23 de dezembro de 2011. Institui a Rede de Atenção Psicossocial para pessoas com sofrimento ou transtorno mental e com necessidades decorrentes do uso de crack, álcool e outras drogas, no âmbito do Sistema Único de Saúde.

Brito, A. A.C., Bonfada, D., e Guimarães, G. (2015). Onde a reforma ainda não chegou: ecos da assistência às urgências psiquiátricas. Physis: Revista de Saúde Coletiva, 25 (4), 1293-1312.
Brito, M., Ramos, M. A., Arruda, V., Dias, A., e Silva, G. B. (2013). Percepção Da Equipe Multiprofissional Do SAMU Frente Às Emergências Psquiátricas. Revista Piauiense de Saúde, 2 (1), 1-11. Disponível em: http://www.revistarps.com.br/index.php/rps/article/ view/11/22

Buedo, P. E., \& Fiadaron, R. (2016). Descripción de las Internaciones en Salud Mental de una Institución Monovalente de la Ciudad de Bahía Blanca, Argentina. Revista de la Asociación Médica de Bahía Blanca, 26 (2), 36-42.

Campos, P. J. (2015). A Rede de Atenção Psicossocial (RAPS) e os Pontos Estratégicos na Atenção à Crise e Urgência. In M.T. Zeferino, J. Rodrigues e J. T. Assis (Orgs), Crise e Urgência em saúde mental: Organização da Atenção Psicossocial à crise e o cuidado em rede (pp. 11-25). Florianópolis: Ministério da Saúde.

Coneglian, L. G., Cavalcante, F. G., e Leandro, J. A. (2015). Reforma psiquiátrica e cidadania: considerações a partir de uma experiência. Revista Trivium - Estudos Interdisciplinares, 7 (1), 127-133. Doi: 10.18370/21764891.2015v1p127

Cortes, J, M. (2016). Lugar de morar: o cotidiano de pessoas com transtornos mentais em um serviço residencial terapêutico. (Tese de Doutoramento). Escola de Enfermagem da Universidade de São Paulo, Brasil.

Daggenvoorde, T., Gijsman, H., \& Goossens, P. (2017). Emergency care in case of acute psychotic and/or manic symptoms: Lived experiences of patients and their families with the first interventions of a mobile crisis team. A phenomenological study. Perspectives in Psychiatric Care, 54 (4), 462-468. Doi:10.1111/ppc. 12247

Demarco, D., Jardim, V., e Kantorski, L. P. (2016). Cuidado em saúde às pessoas com transtorno mental na rede de atenção psicossocial. Revista Cuidado é Fundamental, 8 (3), 4821-4825.

Fernandes, M. A., Pereira, R. F., Leal, M. S., Sales, J. M., e Silva, J. (2016). Cuidados de enfermagem ao paciente psiquiátrico na urgência de um hospital geral. Revista de Enfermagem da Universidade Federal do Piauí, 5 (2), 41- 45. Disponível em: http://www.ojs.ufpi.br/index.php/reufpi/article/view/5241 
Holanda, E., Eduardo, L., Silva, L., Nascimento, R., e Flavio, F. (2016). Atuação Do Enfermeiro em Emergências Psiquiátricas. In: II Congresso Brasileiro de Ciências da Saúde, Campina Grande: Realize

Maftum, M., Silva, Â., Borba, L., Brusamarello, T., e Czarnobay, J. (2017). Mudanças ocorridas na prática profissional na área da saúde mental frente à reforma psiquiátrica brasileira na visão da equipe de enfermagem. Revista Cuidado é fundamental, 9 (2), 309-314. Doi:10.9789/2175-5361.2017.v9i2.309-314

Martins, H., Neto, R., Neto, A. e Velasco, I. (2015). Emergências Clínicas: Abordagem Prática. São Paulo: Manole

Ministério da Saúde. (2013). Cadernos de Atenção Básica, n 34 . Brasília: Secretaria de Atenção à Saúde. Ministério da Saúde

Ministério da Saúde. (2016). Saúde Mental no SUS: Cuidado em Liberdade, Defesa de Direitos e Rede de Atenção Psicossocial. Relatório de Gestão 2011-2015. Disponível em: http://portalarquivos2.saude.gov. br/images/pdf/2016/junho/27/Relat--rio-Gest-o-2011-2015---.pdf

Oliveira, L. e Silva, R. (2017). Saberes e práticas em urgências e emergências psiquiátricas. Revista de Enfermagem da Universidade Estadual do Rio de Janeiro, 25, 1-6. Doi: 10.12957/reuerj.2017.10726

Oliveira, S., Ferreira, W., Vasconcelos, C. e Dutra, D. (2017). O enfrentamento da equipe de enfermagem em atendimentos a pacientes em crise psicótica. Revista de Atenção à Saúde, 15 (53), 50-56. Doi: 10.13037/ras. vol15n53.4598

Paes, M. e Maftum, M. (2013). Percepções da equipe de enfermagem de um pronto atendimento sobre a pessoa com transtorno mental. Revista de Enfermagem da Universidade de Santa Maria, 3 (3), 461-469. Doi: $10.5902 / 217976929852$

Quevedo, J. e Carvalho, A. (2014). Emergências Psiquiátricas [Recursos eletrônicos]. Porto Alegre: Artmed.

Sadock, B., Sadock,V. e Ruiz P. (2016). Compêncio de psiquiatria: Ciência do comportamento e psiquiatria clínica. ( $\left.11^{\mathrm{a}} \mathrm{ed}\right)$. Porto Alegre: Artmed.
Salles, M. e Barros, S. (2013). Representações sociais de usuários de um Centro de Atenção Psicossocial e pessoas de sua rede sobre doença mental e inclusão social. Revista Saúde e sociedade, 22 (4), 1059-1071. Doi: 10.1590/S0104-12902013000400009

Santos, A., Nascimento, Y., Lucena, T., Rodrigues, P., Brêda, M. e Santos, G. (2014). Serviço De Atendimento Móvel De Urgência Às Urgências E Emergências Psiquiátricas. Revista de Enfermagem da Universidade Federal do Pernambuco, 8 (6), 1586-1596. Doi:10.5205/ reuol.5876-50610-1-SM.0806201418

Santos, J. (2013). O estigma da doença mental: compreensão e ações dos trabalhadores do CAPS. São Paulo: Escola de Enfermagem da Universidade de São Paulo.

Silva, M. L., e Dimenstein, M. D. (2014). Manejo da crise: encaminhamento e internação Psiquiátrica em questão. Arquivos Brasileiros de Psicologia, 66 (3), 31 46. Disponível em: http://pepsic.bvsalud.org/pdf/arbp/ v66n3/04.pdf

Willrich, J. (2016). A crise psíquica no discurso da Atenção Psicossocial: um acontecimento de saber, poder e subjetivação. (Tese de Doutoramento). Universidade Federal de Pelotas, Brasil.

Willrich, J., Kantorski, L., Antonacci, M., Cortes, J. e Chiavagatti, F. (2014). Da violência ao vínculo: construindo novos sentidos para a atenção à crise. Revista Brasileira de Enfermagem, 67 (1), 97-103. Doi: 10.5935/0034-7167.20140013

Willrich, J., Kantorski, L., Chiavagatti, F., Cortes, J. e Antonacci, M. (2013). Os sentidos construídos na atenção à crise no território: $O$ centro de atenção psicossocial como protagonista. Revista Escola de Enfermagem da USP, 47 (3), 657-663. Doi:10.1590/ S0080-623420130000300019

Zeller, S. \& Citrome, L. (2016). Managing Agitation Associated with Schizophrenia and Bipolar Disorder in the Emergency Setting. Western Journal of Emergency Medicine, 17 (2), 165-172. Doi:10.5811/ westjem.2015.12.28763 